Pacific Journal of Mathematics

ON INVARIANT SUBSPACES OF SEVERAL VARIABLE 


\title{
ON INVARIANT SUBSPACES OF SEVERAL VARIABLE BERGMAN SPACES
}

\author{
Mihai Putinar
}

\begin{abstract}
By using a natural localization method, one describes the finite codimensional invariant subspaces of the Bergman $n$-tuple of operators associated to some bounded pseudoconvex domains in $\mathbb{C}^{n}$, with a sufficiently nice boundary.
\end{abstract}

0. Introduction. Some recent investigations have been concerned with the structure and classification of the invariant subspaces of the Bergman $n$-tuple of operators, cf. Agrawal-Salinas [2], Axler-Bourdon [4], Bercovici [5], Douglas [7], Douglas-Paulsen [8]. Due to the richness of this lattice of invariant subspaces, the additional assumption on finite codimension was naturally adopted by the above mentioned authors as a first step towards a better understanding of its properties.

The present note arose from the observation that, when the $L^{2}$ bounded evaluation points of a pseudoconvex domain lie in the Fredholm resolvent set of the associated Bergman $n$-tuple, then the description of finite codimensional invariant subspaces is, at least conceptually, a fairly simple algebraic matter. This simplification requires only the basic properties of the sheaf model for systems of commuting operators introduced in [11].

The main result below is also available by some other recent methods. First is the quite similar technique of localizing Hilbert modules over function algebras, due to Douglas [7] and Douglas and Paulsen [8], and secondly is the study of the so-called canonical subspaces of some Hilbert spaces with reproducing kernels, developed by Agrawal and Salinas [2]. Both points of view will be discussed in $\S 2$ of this note.

In fact the Bergman space of a pseudoconvex domain is only an example within a class of abstract Banach $\mathscr{O}\left(\mathbb{C}^{n}\right)$-modules, whose finite codimensional submodules turn out to have a similar structure. The precise formulation of this remark ends the note.

We would like to thank the referee, whose observations pointed out some bibliographical omissions in a first version of the manuscript.

1. Pseudoconvex domains. Let $\Omega$ be a bounded pseudoconvex domain in $\mathbb{C}^{n}, n \geq 1$, and let $L_{a}^{2}(\Omega)$ denote the corresponding Bergman 
space, i.e. the Hilbert space of all holomorphic and square summable functions defined on $\Omega$. The $n$-tuple $M_{\Omega}=\left(z_{1}, \ldots, z_{n}\right)$ of multiplication operators on $L_{a}^{2}(\Omega)$ by the corresponding coordinate functions is referred to as the Bergman $n$-tuple of $\Omega$.

In this section we isolate a class of pseudoconvex domains which will be convenient for our techniques. A similar and deeper analysis is carried out in [4], in the case of one complex variable.

In the sequel $\mathscr{O}$ denote the sheaf of complex analytic functions on $\mathbb{C}^{n}$.

LEMMA 1. Let $\Omega$ be a bounded pseudoconvex domain in $\mathbb{C}^{n}$ and let $\lambda \in \partial \Omega$. Assume that $\bar{\Omega}$ is a Stein compact and that $\mathscr{O}(\bar{\Omega})$ is dense in $L_{a}^{2}(\Omega)$. Then the following assertions are equivalent:

(i) The subspace $\sum_{j=1}^{n}\left(z_{j}-\lambda_{j}\right) L_{a}^{2}(\Omega)$ is dense in $L_{a}^{2}(\Omega)$.

(ii) There is no positive constant $C$, such that

$$
|f(\lambda)| \leq C\|f\|_{2, \Omega}
$$

for every function $f \in \mathscr{O}(\overline{\mathbf{\Omega}})$.

It is quite obvious that conditions (i) and (ii) are also equivalent to the spectral property $\lambda \notin \sigma_{p}\left(M_{\Omega}^{*}\right)$, where $\sigma_{p}$ denotes the joint point spectrum.

Proof. (i) $\Rightarrow$ (ii). Assume that there exists a constant $C>0$, so that the estimate $(1)$ holds. Then $\lim _{m \rightarrow \infty} f_{m}(\lambda)=: f(\lambda)$ exists for every convergent sequence $f_{m} \rightarrow f, f_{m} \notin \mathscr{O}(\bar{\Omega}), f \in L_{a}^{2}(\Omega)$.

If assertion (i) would be true, then $f(\lambda)=0$ for every element $f \in L_{a}^{2}(\Omega)$, which is evidently a contradiction.

(ii) $\Rightarrow(\mathrm{i})$. Assume that there exists a function $g \in L_{a}^{2}(\Omega), g \neq 0$, which is orthogonal to the subspace $\sum_{j=1}^{n}\left(z_{j}-\lambda_{j}\right) L_{a}^{2}(\Omega)$. Let $g_{m} \in$ $\mathscr{O}(\bar{\Omega})$ be a sequence which approximates $g$ in the norm of $L_{a}^{2}(\Omega)$. In view of the hypothesis on $\bar{\Omega}$ to possess a fundamental system of open pseudoconvex neighbourhoods, every function $g_{m}$ can be decomposed as follows:

$$
g_{m}(z)=g_{m}(\lambda)+\sum_{j=1}^{n}\left(z_{j}-\lambda_{j}\right) g_{m}^{j}(z),
$$

where $g_{m}^{j} \in \mathscr{O}(\bar{\Omega}), 1 \leq j \leq n, 1 \leq m$. This is possible by a standard application of Cartan's Theorem B. Accordingly

$$
\left\langle g_{m}, g\right\rangle=g_{m}(\lambda)\langle 1, g\rangle,
$$

whence we infer by passing to the limit $m \rightarrow \infty$, that $\langle 1, g\rangle \neq 0$. 
Let $f \in \mathscr{O}(\bar{\Omega})$. By arguing as above we obtain

$$
|f(\lambda)\langle 1, g\rangle|=|\langle f, g\rangle| \leq\|f\| \cdot\|g\|,
$$

which proves that assertion (ii) is not true.

The above lemma was intended to bring forward the following classes of examples.

EXAMPLE 1. A boundary point of a strictly pseudoconvex domain with smooth boundary satisfies condition (ii) above.

Indeed, let $\Omega$ be a strictly pseudoconvex domain with smooth boundary and let $\lambda \in \partial \Omega$ be fixed. Assume by way of contradiction that there exists a constant $C>0$, such that the estimate (1) holds for $\lambda$. Then repeating an argument given in the previous proof,

$$
f(\lambda)=\lim _{m \rightarrow \infty} f_{m}(\lambda)
$$

exists whenever $f_{m} \rightarrow f$ in $L_{a}^{2}(\Omega)$ and $f_{m} \in \mathscr{O}(\overline{\mathbf{\Omega}})$. Moreover, in this case relation (1) holds for $f$. By Theorem 3.4.9 of [10], the algebra $\mathscr{O}(\bar{\Omega})$ is dense in $L_{a}^{2}(\Omega)$. In particular any element of $\mathscr{A}^{\infty}(\Omega)=\mathscr{O}(\Omega) \cap \mathscr{C}^{\infty}(\Omega)$ is approximable in the $L^{2}(\Omega)$-norm by functions belonging to $\mathscr{O}(\overline{\boldsymbol{\Omega}})$.

Since $\lambda$ is a peak point for the algebra $\mathscr{A}^{\infty}(\Omega)$, see for instance [6], there exists $h \in \mathscr{A}^{\infty}(\Omega)$ with the properties $h(\lambda)=1$ and $|h(z)|<1$ for $z \in \bar{\Omega} \backslash\{\lambda\}$. Then

$$
1 \leq C\left\|h^{m}\right\|_{2, \Omega}
$$

for every natural $m$, and $\lim _{m \rightarrow \infty}\left\|h^{m}\right\|_{2, \Omega}=0$. This contradicts our assumption and thus condition (ii) is verified.

EXAMPLE 2 . Let $\Omega=\Omega_{1} \times \cdots \times \Omega_{n}$ be a polydomain whose factors $\Omega_{j}$ satisfy the following requirement: no connected component of $\partial \Omega_{j}$ is reduced to a point, $1 \leq j \leq n$. Then every point $\lambda \in \partial \Omega$ verifies condition (ii).

In order to prove this fact we need the identification $L_{a}^{2}(\Omega)=$ $L_{a}^{2}\left(\Omega_{1}\right) \tilde{\otimes} \cdots \tilde{\otimes} L_{a}^{2}\left(\Omega_{n}\right)$, where “ $\tilde{\otimes}$ " denotes the hilbertian tensor product.

By virtue of Theorem 5 of [4], the subspace $\left(z_{j}-\lambda_{j}\right) L_{a}^{2}\left(\Omega_{j}\right)$ is dense in $L_{a}^{2}\left(\Omega_{j}\right)$ for every $\lambda_{j}$ in $\partial \Omega_{j}$. 
Fix a point $\lambda \in \partial \Omega$. Since at least one of its entries $\lambda_{j}$ belongs to $\partial \Omega_{j}$, we get

$$
\begin{aligned}
& {\left[\sum_{j=1}^{n}\left(z_{j}-\lambda_{J}\right) L_{a}^{2}(\Omega)\right]^{-}} \\
& \quad \supset\left[L_{a}^{2}\left(\Omega_{1}\right) \tilde{\otimes} \cdots \tilde{\otimes}\left(z_{J}-\lambda_{j}\right) L_{a}^{2}\left(\Omega_{j}\right) \tilde{\otimes} \cdots \tilde{\otimes} L_{a}^{2}\left(\Omega_{n}\right)\right]^{-}=L_{a}^{2}(\Omega) .
\end{aligned}
$$

By an inspection of the proof of the previous lemma it follows that the implication (i) $\Rightarrow$ (ii) remains valid without any additional assumption on $\Omega$. Therefore condition (ii) is verified for the point $\lambda \in \partial \Omega$.

EXAMPLE 3. Any bounded pseudoconvex Reinhardt domain of $\mathbb{C}^{n}$ satisfies (i) and (ii).

Let $\Omega \subset \mathbb{C}^{n}$ be a bounded pseudoconvex Reinhardt domain with the associated Bergman kernel $K$. It is easy to remark that each of the conditions (i) or (ii) is in that case equivalent to $K(\lambda, \lambda)=\infty$.

Definition 1. Let $\mathscr{C}$ denote the class of those bounded pseudoconvex domains $\Omega$ in $\mathbb{C}^{n}$, which fulfill the following condition: for every point $\lambda$ in $\partial \Omega$ there is no constant $C>0$ with the property:

$$
|f(\lambda)| \leq C\|f\|_{2, \Omega}, \quad f \in \mathscr{O}(\Omega) .
$$

The preceding examples provide elements of $\mathscr{C}$. Also it is worth mentioning that the class $\mathscr{C}$ is closed under cartesian products and analytic isomorphisms which extend to the boundary.

2. The main result. In complete analogy with the first part of [4] we can state the next.

THEOREM 1. Let $\Omega$ be a domain belonging to the class $\mathscr{C}$. Any $M_{\Omega^{-}}$invariant subspace $S$ of finite codimension in $L_{a}^{2}(\Omega)$ has the form

$$
S=\sum_{j=1}^{k} P_{j} L_{a}^{2}(\Omega),
$$

where $P_{j}$ are polynomials having a finite number of common zeros, all contained in $\Omega$.

Actually we are in the position to discuss at least two different ways of proving Theorem 1. Some terminology and facts needed in the first proof below will be recalled in $\S 3$, in connection with a generalization of Theorem 1. 
Proof 1. Consider an invariant subspace $S$ as in the statement and denote $Q=L_{a}^{2}(\Omega) / S$. Instead of working with $n$-tuples of commuting operators, we adopt the equivalent point of view of $\mathscr{O}\left(\mathbb{C}^{n}\right)$ modules.

It was proved in [11], as a byproduct of Hörmander's $L^{2}$-estimates for the $\bar{\partial}$-operator, that $L_{a}^{2}(\Omega)$ is a module with property $(\beta)$. Roughly speaking, that means that $L_{a}^{2}(\Omega)$ is suitable for localization in the category of Fréchet $\mathscr{O}\left(\mathbb{C}^{n}\right)$-modules, see $\S 3$ below for details.

Since $\operatorname{dim} Q<\infty$, the module $Q$ also has property $(\beta)$. From the exact sequence

$$
0 \rightarrow S \rightarrow L_{a}^{2}(\Omega) \rightarrow Q \rightarrow 0
$$

one deduces that $S$ has the same property, too. Let us denote by $\mathscr{S}$, $\mathscr{F}, \mathscr{Q}$ the corresponding Fréchet quasi-coherent $\mathscr{O}$-modules, see $\S 3$. They are related by a similar exact sequence

$$
0 \rightarrow \mathscr{S} \rightarrow \mathscr{F} \rightarrow \mathscr{Q} \rightarrow 0 .
$$

Recall that $\mathscr{F}$ was called in [11] the sheaf model of the system of operators $M_{\Omega}$.

Among other things, it was proved in $\S I V$ of [11] that there exists an exact sequence of Fréchet $\mathscr{O}$-modules, derived from the Dolbeault complex:

$$
0 \rightarrow \mathscr{F} \rightarrow \mathscr{M}^{0} \rightarrow \mathscr{M}^{1} \rightarrow \cdots \rightarrow \mathscr{M}^{n} \rightarrow 0
$$

with $\left.\left.\mathscr{M}^{j}\right|_{\Omega} \cong \mathscr{E}^{(0, j)}\right|_{\Omega}$, where $\mathscr{E}^{(0, j)}$ stands for the sheaf of smooth differential forms of bidegree $(0, j), 0 \leq j \leq n$. Consequently, $\left.\left.\mathscr{F}\right|_{\Omega} \cong \mathscr{O}\right|_{\Omega}$.

The last isomorphism can equally be obtained by a direct computation on the Koszul complex of $M_{\Omega}$.

Let $\lambda \in \operatorname{Supp}(\mathscr{Q})$ and denote by $\mathfrak{m}_{\lambda}$ the sheaf of ideals of $\mathscr{Q}$, consisting of those analytic functions which vanish at $\lambda$. Because $\mathscr{O} / \mathfrak{m}_{\lambda}$ is a quotient module of $\mathscr{Q}$, at the level of global sections there exists a continuous $\mathscr{O}\left(\mathbb{C}^{n}\right)$-linear map:

$$
\varepsilon_{\lambda}: L_{a}^{2}(\Omega) \rightarrow \mathbb{C},
$$

with the property $\varepsilon_{\lambda}(f)=f(\lambda)$ for every function $f \in \mathscr{O}(\bar{\Omega})$. By taking into account the hypothesis $\Omega \in \mathscr{C}$ one gets $\lambda \in \Omega$. In conclusion we have proved the inclusion $\operatorname{Supp}(\mathscr{Q}) \subset \Omega$.

As a finite length module, $\mathscr{Q}$ is even a module over the algebra of polynomials. Hence by Hilbert's Syzygies Theorem it admits a finite resolution of the form:

$$
0 \rightarrow \mathscr{O}^{r} \rightarrow \cdots \rightarrow \mathscr{O}^{k} \stackrel{\left(P_{1}, \ldots, P_{k}\right)}{\longrightarrow} \mathscr{O} \rightarrow \mathscr{Q} \rightarrow 0,
$$


where $P_{j}$ are polynomials. Moreover, $P_{1}(\lambda)=\cdots=P_{k}(\lambda)=0$ if and only if $\lambda \in \operatorname{Supp}(\mathscr{Q})$.

Since the sheaf $\mathscr{F}$ is isomorphic to $\mathscr{O}$ in a neighbourhood of $\operatorname{Supp}(\mathscr{Q})$, one finds $\mathscr{Q} \otimes_{\mathscr{Q}} \mathscr{F} \cong \mathscr{Q}$ and $\operatorname{Tor}_{p}^{\mathscr{Q}}(\mathscr{Q}, \mathscr{F})=0$ for $p>0$. Accordingly, the sequence (2) remains exact after tensor multiplication with $\mathscr{F}$ :

$$
0 \rightarrow \mathscr{F}^{r} \rightarrow \cdots \rightarrow \mathscr{F}^{k} \stackrel{\left(P_{1}, \ldots, P_{k}\right)}{\longrightarrow} \mathscr{F} \rightarrow \mathscr{Q} \rightarrow 0 .
$$

But the sheaf $\mathscr{F}$ is acyclic on $\mathbb{C}^{n}$, so that by passing to global sections one finally obtains the exact sequence:

$$
0 \rightarrow\left[L_{a}^{2}(\Omega)\right]^{r} \rightarrow \cdots \rightarrow\left[L_{a}^{2}(\Omega)\right]^{k} \stackrel{\left(P_{1}, \ldots, P_{k}\right)}{\longrightarrow} L_{a}^{2}(\Omega) \rightarrow Q \rightarrow 0 .
$$

This completes the first proof of Theorem 1.

Proof 2. Let us assume for simplicity that the algebra $\mathscr{O}(\bar{\Omega})$ is dense in $L_{a}^{2}(\Omega)$. Let $S \subset L_{a}^{2}(\Omega)$ be a finite codimensional $M_{\Omega}$-invariant subspace. Then the natural restriction and projection map:

$$
\rho: \mathscr{O}(\overline{\mathbf{\Omega}}) \rightarrow L_{a}^{2}(\Omega) / S
$$

is onto because $\mathscr{O}(\bar{\Omega})$ is dense in $L_{a}^{2}(\Omega)$ and $\operatorname{dim} L_{a}^{2}(\Omega) / S<\infty$.

If one denotes $I=\operatorname{Ker} \rho$, then $I$ is a finite codimension ideal of $\mathscr{O}(\bar{\Omega})$, with the property that its $L^{2}(\Omega)$-closure $I^{-}$is contained in $S$. Let $L$ be a linear complement of $I$ in $O(\bar{\Omega})$. Since

$$
L_{a}^{2}(\Omega)=O(\bar{\Omega})^{-}=I^{-}+L,
$$

we infer that $\operatorname{dim}\left(L_{a}^{2}(\Omega) / I^{-}\right)=\operatorname{dim}(\mathscr{O}(\bar{\Omega}) / I)$, whence $S=I^{-}$. This argument was adapted after Ahern and Clark [3].

In view of the assumption on $\Omega$ to belong to the class $\mathscr{C}$, the quotient module $\mathscr{O}(\bar{\Omega}) / I$ is supported by a finite subset of $\Omega$. Hence, by repeating for instance the argument given in Proof 1 ,

$$
I=\left(P_{1}, \ldots, P_{k}\right) \mathscr{O}(\bar{\Omega}),
$$

where $P_{1}, \ldots, P_{k}$ are polynomials. Therefore

$$
S=\left(\sum_{j=1}^{k} P_{j} L_{a}^{2}(\Omega)\right)^{-}
$$

At this moment the fact that the submodule $\sum_{j=1}^{k} P_{j} L_{a}^{2}(\Omega)$ is actually closed can be proved at least in two different ways. 
First, this assertion follows as in Proof 1 by means of a localization procedure, this time in the category of Hilbert $O(\Omega)$-modules, cf. Douglas and Paulsen [8].

Or secondly, one may apply Agrawal and Salinas' analysis of the analytic subspaces of the Bergman space [2]. Quite specifically, it turns out that $P_{1} L_{a}^{2}(\Omega)+\cdots+P_{k} L_{a}^{2}(\Omega)$ is a finite dimensional perturbation of a "canonical subspace", see [2] Theorems 2.8 and 3.11, and consequently it is closed.

REMARKS. (a) Theorem 1 establishes a one-to-one correspondence between the $M_{\Omega}$-invariant subspaces $S$ of finite codimension in $L_{a}^{2}(\Omega)$ and the ideals $I \subset \mathbb{C}\left[z_{1}, \ldots, z_{n}\right]$, with the property that the natural restriction map:

$$
C\left[z_{1}, \ldots, z_{n}\right] / I \rightarrow L_{a}^{2}(\Omega) / S
$$

is an isomorphism.

Moreover, the last part of Proof 1 shows that

$$
S \otimes_{\mathscr{O}\left(\mathbb{C}^{n}\right)} N=\left(I \cdot L_{a}^{2}(\Omega)\right) \otimes_{\mathscr{O}\left(\mathbb{C}^{n}\right)} N=(I \cdot \mathscr{O}(\Omega)) \otimes_{\mathscr{O}(\Omega)} N=I \cdot N,
$$

whenever $N$ is a finite codimensional $\mathscr{O}\left(\mathbb{C}^{n}\right)$-module supported by $\Omega$. As Douglas and Paulsen recently discovered, the existence of such isomorphisms insures two finite codimensional $\mathscr{O}\left(\mathbb{C}^{n}\right)$-submodules of $L_{a}^{2}(\Omega)$ to be quasi-similar if and only if they are equal, see [8, Chapter $6]$.

Thus the classification of the submodules of the Bergman space described in Theorem 1 turns out to be a purely algebraic problem.

(b) By comparing the exact sequences (2) and (3) one finds that the only relations between the generators $P_{1}, \ldots, P_{k}$ of the finite codimensional subspaces $S \subset L_{a}^{2}(\Omega)$ are the algebraic ones, and so on for the iterated relations.

(c) It was pointed out in [4], and the same conclusion remains valid for any $n>1$, that there exist domains $\Omega \subset \mathbb{C}^{n}$ not belonging to the class $\mathscr{C}$, for which the conclusion of Theorem 1 fails to be true.

3. Extension to an abstract setting. The essential property of the Bergman space needed in the Proof 1 of Theorem 1 was the existence of a $\bar{\partial}$-resolution with Hilbert spaces of differential forms. It is exactly this property which characterizes the Fréchet $\mathscr{O}\left(\mathbb{C}^{n}\right)$-modules which are well fitted for localization. To be more precise we first need some terminology. 
Let $X$ and $Y$ be Fréchet modules over the algebra of entire functions $\mathscr{O}\left(\mathbb{C}^{n}\right)$. One denotes after J. Taylor [13]:

$$
X \hat{\otimes}_{\mathscr{O}\left(\mathbb{C}^{n}\right)} Y=\operatorname{Coker} \delta,
$$

where $\delta: X \hat{\otimes} \mathscr{O}\left(\mathbb{C}^{n}\right) \hat{\otimes} Y \rightarrow X \hat{\otimes} Y, \delta(x \otimes f \otimes y)=x f \otimes y-x \otimes f y$, where $x \in X, y \in Y, f \in \mathscr{O}\left(\mathbb{C}^{n}\right)$ and " $\hat{\otimes}$ " is a complete topological tensor product, as for instance the projective one. Under some natural nuclearity or splitting conditions (fulfilled in our case), the above relative tensor product admits derived functors, denoted $\widehat{\text { Tor }}$, see [13].

Let $X$ be a Banach $\mathscr{O}\left(\mathbb{C}^{n}\right)$-module. The following assertions are equivalent:

(a) There exists a Fréchet $\mathscr{O}$-module $\mathscr{F}$ defined on $\mathbb{C}^{n}$, such that $X=\mathscr{F}\left(\mathbb{C}^{n}\right), \mathscr{F}$ is acyclic on Stein open subsets of $\mathbb{C}^{n}$ and $\mathscr{F}$ admits locally resolutions to the left with topologically free $\mathscr{O}$-modules (i.e. of the form $\mathscr{O} \hat{\otimes} E$ with a Fréchet space $E$ );

(b) For every polydisk $\Delta \subset \mathbb{C}^{n}$, the locally convex space $X \hat{\otimes}_{\mathscr{O}\left(\mathbb{C}^{n}\right)} \mathscr{O}(\Delta)$ is separated and $\widehat{\operatorname{Tor}}_{p}^{\mathscr{O}\left(\mathbb{C}^{n}\right)}(X, \mathscr{O}(\Delta))=0$ for any $p \geq 1$;

(c) There exists a finite resolution to the right with Banach $\mathscr{O}\left(\mathbb{C}^{n}\right)$ modules:

$$
0 \rightarrow X \rightarrow \mathscr{D}^{0}\left(\mathbb{C}^{n}\right) \rightarrow \cdots \rightarrow \mathscr{D}^{N}\left(\mathbb{C}^{n}\right) \rightarrow 0,
$$

where $\mathscr{D}^{j}$ are Fréchet soft $\mathscr{O}$-modules, $0 \leq j \leq N$. For a proof see [11] and [12].

When referring to the associated $n$-tuple $T=\left(T_{1}, \ldots, T_{n}\right)$ of commuting operators $\left(T_{j} x=z_{j} x, x \in X, 1 \leq j \leq n\right)$, condition (b) is known as Bishop's property $(\beta)$, and has a long history.

The Fréchet $\mathscr{O}$-modules appearing in condition (a) were called quasicoherent by the French school of modern geometry. They are a powerful tool in the study of singular analytic spaces; see [11] for further references.

The equivalence $(\mathrm{a}) \Leftrightarrow(\mathrm{b})$ characterizes the $n$-tuples of commuting operators with property $(\beta)$ as those $n$-tuples which admit a Fréchet quasi-coherent sheaf model. On the other hand, the equivalence (b) $\Leftrightarrow$ (c) is the characterization of property $(\beta)$ by the existence of an abstract $\bar{\partial}$-resolution.

To give only a class of examples, close to the spirit of the present note, let $\Omega$ be a bounded domain of $\mathbb{C}^{n}$, with smooth strictly pseudoconvex boundary. Let also fix a number $p \in[1, \infty]$. The $L^{p}$ estimates for the $\bar{\partial}$-operator on $\Omega$, see for instance [9], yield 
similarly to the Bergman space case an exact sequence of Banach $\mathscr{O}\left(\mathbb{C}^{n}\right)$-modules:

$$
0 \rightarrow L_{a}^{p}(\Omega) \rightarrow D^{0} \rightarrow \cdots \rightarrow D^{n} \rightarrow 0
$$

where $L_{a}^{p}(\Omega)=L^{p}(\Omega) \cap \mathscr{O}(\Omega)$ and $D^{j}$ are some Banach $\mathscr{C}^{\infty}\left(\mathbb{C}^{n}\right)$ modules of differential forms.

Thus, according to the equivalence (b) $\Leftrightarrow(\mathrm{c})$, the $\mathscr{O}\left(\mathbb{C}^{n}\right)$-module $L_{a}^{p}(\Omega)$ has property $(\beta)$. Its sheaf model $\mathscr{F}$ has also a simple description, [12]:

$$
\mathscr{F}(U)=\left\{f \in \mathscr{O}(U \cap \Omega) ;\|f\|_{p, K}<\infty \text { for } K \subset \subset U\right\}
$$

where $U$ is an open subset of $\mathbb{C}^{n}$.

Other examples of $\mathscr{O}\left(\mathbb{C}^{n}\right)$-modules with property $(\beta)$ are the finite dimensional modules or the global sections of Fréchet soft $\mathscr{O}$-modules, [11].

The same lines of the Proof 1 above can be used in order to obtain the following generalization of Theorem 1.

Proposition 1. Let $T$ be a commutative $n$-tuple of operators with property $(\beta)$ acting on the Banach space $X$. Assume that for every $\lambda \in \sigma(T)$, either

$$
\sum_{j=1}^{n}\left(T_{j}-\lambda_{j}\right) X
$$

is dense in $X$, or

$$
\operatorname{dim}\left(X / \sum_{j=1}^{n}\left(T_{j}-\lambda_{j}\right) X\right)=1
$$

Then every $T$-invariant subspace $S$ of finite codimension in $X$ has the form

$$
S=\sum_{i=1}^{k} P_{i}(T) X,
$$

where $P_{i}$ are polynomials having only a finite number of zeros, all lying in $\sigma(T) \backslash \sigma_{\text {ress }}(T)$.

The spaces $L_{a}^{p}(\Omega), 1 \leq p \leq \infty$, described above verify the hypothesis of Proposition 1; hence the same conclusion holds for all of them, similarly to the Bergman space case. 
Of course Proposition 1 does not exhaust the possible examples of Banach $\mathscr{O}\left(\mathbb{C}^{n}\right)$-modules possessing a similar lattice of finite codimensional submodules.

\section{REFERENCES}

[1] O. P. Agrawal, D. N. Clark, and R. G. Douglas, Invariant subspaces in the polydisk, Pacific J. Math., 121 (1986), 1-11.

[2] O. P. Agrawal and N. Salinas, Sharp kernels and canonical subspaces, Amer. J. Math., 109 (1987), 23-48.

[3] P. R. Ahern and D. N. Clark, Invariant subspaces and analytic continuation in several variables, J. Math. Mech., 19 (1970), 963-969.

[4] S. Axler and P. Bourdon, Finite codimensional invariant subspaces of Bergman spaces, Trans. Amer. Math. Soc., 306: 2 (1988), 805-817.

[5] H. Bercovici, A question on invariant subspaces of Bergman spaces, Proc. Amer. Math. Soc., 103 (1988), 759-760.

[6] J. Chaumat and A. M. Chollet, Charactérisation et propriétés des ensembles localement pic de $A^{\infty}(D)$, Duke Math. J., 47 (1980), 763-787.

[7] R. G. Douglas, On Shilov resolutions of Hilbert modules, in the volume "Special Classes of Linear Operators and Other Topics”, pp. 51-60, Birkhäuser, BaselBoston-Stuttgart, 1988.

[8] R. G. Douglas and V. I. Paulsen, Hilbert Modules over Function Algebras, Pitman Research Notes in Math., vol. 217, Longman, London, 1989.

[9] G. Henkin and J. Leiterer, Theory of Functions on Complex Manifolds, Akademie-Verlag, Berlin, 1984.

[10] L. Hörmander, $L^{2}$-estimates and existence theorems for the $\bar{\partial}$-operator, Acta Math., 113 (1-2) (1965), 89-152.

[11] M. Putinar, Spectral theory and sheaf theory. II, Math. Z., 192 (1986), 473-490.

[12] __ Spectral theory and sheaf theory. IV, Proc. Symp. Pure Math., 51 (1990).

[13] J. L. Taylor, Homology and cohomology for topological algebras, Adv. in Math., 9 (1972), 131-182.

Received November 9, 1988 and in revised form January 2, 1990.

INSTITUTE OF MATHEMATICS

BDUl PACII 220

79622 BUCHAREST, ROMANIA

Current address: University of Kansas

Lawrence, Kansas 66045 


\title{
PACIFIC JOURNAL OF MATHEMATICS EDITORS
}

\author{
V. S. VARADARAJAN \\ (Managing Editor) \\ University of California \\ Los Angeles, CA 90024-1555-05 \\ Herbert Clemens \\ University of Utah \\ Salt Lake City, UT 84112 \\ THOMAS ENRIGHT \\ University of California, San Diego \\ La Jolla, CA 92093
}

R. FINN

Stanford University

Stanford, CA 94305

Hermann FlaschKa

University of Arizona

Tucson, AZ 85721

VAUGHaN F. R. JoNes

University of California

Berkeley, CA 94720

Steven Kerckhoff

Stanford University

Stanford, CA 94305

\section{C. MOORE}

University of California

Berkeley, CA 94720

Martin ScharlemanN

University of California

Santa Barbara, CA 93106

HAROLD STARK

University of California, San Diego

La Jolla, CA 92093
R. ARENS
E. F. BECKENBACH
F. WolF
K. YoshidA

\section{ASSOCIATE EDITORS}

\begin{tabular}{ll}
\multicolumn{1}{c}{ SUD-1982) } & \multicolumn{1}{c}{$(1904-1989)$} \\
UNIVERSITY OF ARIZONA & UNIVERSITY OF OREGON \\
UNIVERSITY OF BRITISH COLUMBIA & UNIVERSITY OF SOUTHERN CALIFORNIA \\
CALIFORNIA INSTITUTE OF TECHNOLOGY & STANFORD UNIVERSITY \\
UNIVERSITY OF CALIFORNIA & UNIVERSITY OF HAWAII \\
MONTANA STATE UNIVERSITY & UNIVERSITY OF TOKYO \\
UNIVERSITY OF NEVADA, RENO & UNIVERSITY OF UTAH \\
NEW MEXICO STATE UNIVERSITY & WASHINGTON STATE UNIVERSITY \\
OREGON STATE UNIVERSITY & UNIVERSITY OF WASHINGTON \\
\hline
\end{tabular}

The Supporting Institutions listed above contribute to the cost of publication of this Journal, but they are not owners or publishers and have no responsibility for its content or policies.

\begin{abstract}
Mathematical papers intended for publication in the Pacific Journal of Mathematics should be in typed form or offset-reproduced (not dittoed), double spaced with large margins. Please do not use built up fractions in the text of the manuscript. However, you may use them in the displayed equations. Underline Greek letters in red, German in green, and script in blue. The first paragraph must be capable of being used separately as a synopsis of the entire paper. In particular it should contain no bibliographic references. Please propose a heading for the odd numbered pages of less than 35 characters. Manuscripts, in triplicate, may be sent to any one of the editors. Please classify according to the 1980 Mathematics Subject Classification (1985 Revision) scheme which can be found in the December index volumes of Mathematical Reviews. Supply name and address of author to whom proofs should be sent. All other communications should be addressed to the managing editor, or Elaine Barth, University of California, Los Angeles, California 90024-1555-05.

There are page-charges associated with articles appearing in the Pacific Journal of Mathematics. These charges are expected to be paid by the author's University, Government Agency or Company. If the author or authors do not have access to such Institutional support these charges are waived. Single authors will receive 50 free reprints; joint authors will receive a total of 100 free reprints. Additional copies may be obtained at cost in multiples of 50 .
\end{abstract}

The Pacific Journal of Mathematics (ISSN 0030-8730) is published monthly except for July and August. Regular subscription rate: $\$ 190.00$ a year (10 issues). Special rate: $\$ 95.00$ a year to individual members of supporting institutions.

Subscriptions, orders for numbers issued in the last three calendar years, and changes of address should be sent to Pacific Journal of Mathematics, P.O. Box 969, Carmel Valley, CA 93924, U.S.A. Old back numbers obtainable from Kraus Periodicals Co., Route 100, Millwood, NY 10546.

The Pacific Journal of Mathematics at P.O. Box 969, Carmel Valley, CA 93924 (ISSN 0030-8730) is published monthly except for July and August. Second-class postage paid at Carmel Valley, California 93924, and additional mailing offices. Postmaster: send address changes to Pacific Journal of Mathematics, P.O. Box 969, Carmel Valley, CA 93924.

PUBLISHED BY PACIFIC JOURNAL OF MATHEMATICS, A NON-PROFIT CORPORATION Copyright (C) 1991 by Pacific Journal of Mathematics 